\title{
The response of the ionosphere to faint and bright solar flares as deduced from global GPS network data
}

Edward L. Afraimovich, Alexandre T. Altynsev, Victor V. Grechnev and Ludmila A. Leonovich Institute of Solar-Terrestrial Physics SD, Russian Academy of Sciences, Irkutsk, Russia

\begin{abstract}
Results derived from analysing the ionosphere response to faint and bright solar flares are presented. The analysis used technology of a global detection of ionospheric effects from solar flares as developed by the authors, on the basis of phase measurements of the Total Electron Content (TEC) in the ionosphere using an international GPS network. The essence of the method is that use is made of appropriate filtering and a coherent processing of variations in the TEC which is determined from GPS data, simultaneously for the entire set of visible GPS satellites at all stations used in the analysis. This technique is useful for identifying the ionospheric response to faint solar flares (of X-ray class C) when the variation amplitude of the TEC response to separate line-on-sight to GPS satellite is comparable to the level of background fluctuations. The dependence of the TEC variation response amplitude on the bright flares location on the Sun is investigated.
\end{abstract}

Key words Sudden Ionospheric Disturbances (SID) - solar flare - GPS

\section{Introduction}

The enhancement of X-ray and ultraviolet (UV) emission observed during chromospheric flares on the Sun immediately causes an increase in electron density in the ionosphere. These density variations differ for different altitudes and are called Sudden Ionospheric Disturbances (SID) (Donnelly, 1969; Davies, 1990). SIDs are generally recorded as the Short Wave Fadeout (SWF) (Stonehocker, 1970); Sudden Phase Anomaly (SPA) (Jones, 1971; Ohshio, 1971); Sudden Frequency Deviation (SFD) (Donnelly,

Mailing address: Prof. Edward L. Afraimovich, Institute of Solar-Terrestrial Physics SD, Russian Academy of Sciences, P.O. Box 4026, Irkutsk, 664033, Russia; e-mail: afra@iszf.ru
1971; Liu et al., 1996); Sudden Cosmic Noise Absorption (SCNA) (Deshpande and Mitra, 1972); Sudden Enhancement/decrease of atmospherics (SES) (Sao et al., 1970). Much research is devoted to SID studies, among them a number of thorough reviews (Mitra, 1974; Davies, 1990).

SFD are caused by an almost time-coincident increase in electron densities in the $E$ - and $F$ region on the dayside of the Earth (Davies, 1990; Donnelly, 1969; Liu et al., 1996). A limitation of this method is the uncertainty in the spatial and altitude localization of the UV flux effect, the inadequate number of paths, and the need to use special-purpose equipment.

The effect of solar flares on the ionospheric $F$-region is also manifested as a Sudden Increase of Total Electron Content (SITEC) which was measured previously using continuously operating VHF radio beacons on geostationary satellites (Mendillo et al., 1974b; Davies, 1980). A serious limitation of methods based on analyzing VHF signals from geostationary 
satellites is their small and ever decreasing (with time) number and the nonuniform distribution in longitude. Hence it is impossible to make measurements in some geophysically interesting regions of the globe, especially in high latitudes.

A further, highly informative, technique is the method of Incoherent Scatter (IS) (Thome and Wagner, 1971; Mendillo et al., 1974a). However, the practical implementation of the IS method requires very sophisticated, expensive equipment. The IS method's time resolution is inadequate for the study of ionospheric effects from solar flares. The time of electron density enhancement in the $E$ and $F_{1}$ regions during impulsive flares can be 2-3 min.

Consequently, none of the above-mentioned existing methods can serve as an effective basis for the radio detection system to provide a continuous, global SID monitoring with adequate space-time resolution. Furthermore, the creation of these facilities requires developing special purpose equipment, including powerful radio transmitters contaminating the radio environment. It is also significant that when using the existing methods, the inadequate spatial aperture gives no way of deducing the possible spatial inhomogeneity of the X-ray and UV flux.

The advent and evolution of a Global Positioning System (GPS) and also the creation on its basis of widely branched networks of GPS stations (at least 900 sites at the August of 2001, the data from which are placed on the Internet) opened up a new era in remote ionospheric sensing. High-precision measurements of the TEC along the Line-Of-Sight (LOS) between the receiver on the ground and transmitters on the GPS system satellites covering the reception zone are made using two-frequency multichannel receivers of the GPS system at almost any point of the globe and at any time simultaneously at two coherently coupled frequencies $f_{1}=1575.42$ $\mathrm{MHz}$ and $f_{2}=1227.60 \mathrm{MHz}$.

The sensitivity of phase measurements in the GPS system is sufficient for detecting irregularities with an amplitude of up to $10^{-3}-10^{-4}$ of the diurnal TEC variation. This makes it possible to detect ionospheric disturbances from different sources of artificial and natural origins. The TEC unit (TECU) which is equal to $10^{16} \mathrm{~m}^{-2}$ and is commonly accepted in the literature, will be used throughout the text.

Afraimovich (2000) and Afraimovich et al. (2000b, 2001a,b) developed a novel technology of a global detection of ionospheric effects from solar flares and presented data from first GPS measurements of global response of the ionosphere to powerful impulsive flares of September 23, 1998, July 29, 1999, and December 28,1999 , were chosen to illustrate the practical implementation of the proposed method. Authors found that fluctuations of TEC, obtained by removing the linear trend of TEC with a time window of about $5 \mathrm{~min}$, are coherent for all stations and LOS on the dayside of the Earth. The time profile of TEC responses is similar to the time behavior of hard X-ray emission variations during flares in the energy range $25-35 \mathrm{keV}$ if the relaxation time of electron density disturbances in the ionosphere of order $50-100 \mathrm{~s}$ is introduced. No such effect on the nightside of the Earth has been detected yet.

The objective of this paper is to use this technology for analysing the ionosphere response to faint and bright solar flares.

\section{Processing of the data from the GPS network}

Following is a brief outline of the global monitoring (detection) technique for solar flares. A physical groundwork for the method is formed by the effect of fast change in electron density in the Earth's ionosphere at the time of a flare simultaneously on the entire sunlit surface. Essentially, the method implies using appropriate filtering and a coherent processing of TEC variations in the ionosphere simultaneously for the entire set of visible (during a given time interval) GPS satellites (as many as 5-10 satellites) at all global GPS network stations used in the analysis.

In detecting solar flares, the ionospheric response is virtually simultaneous for all stations on the dayside of the globe within the time resolution range of the GPS receivers (from $30 \mathrm{~s}$ to $0.1 \mathrm{~s}$ ). Therefore, a coherent processing of TEC variations implies in this case a simple addition of single TEC variations. The detection 
sensitivity is determined by the ability to detect typical signals of the ionospheric response to a solar flare (leading edge duration, period, form, length) at the level of TEC background fluctuations. Ionospheric irregularities are characterized by a power spectrum, so that background fluctuations will always be distinguished in the frequency range of interest.

However, the spatial scale on which the averaging is carried out substantially exceeds the correlation scales of background fluctuations. With a typical length of X-ray bursts and UV emission of solar flares of about 5-10 min, the corresponding ionization irregularity size does normally not exceed $30-50 \mathrm{~km}$; hence the condition of a statistical independence of TEC fluctuations at spaced beams is almost always satisfied. Therefore, coherent summation of responses to a flare on a set of LOS spaced throughout the dayside of the globe permits the solar flare effect to be detected even when the response amplitude on partial LOS is markedly smaller than the noise level (background fluctuations).

The proposed procedure of coherent accumulation is essentially equivalent to the operation of coincidence schemes which are extensively used in X-ray and gamma-ray telescopes. If the SID response and background fluctuations, respectively, are considered to be the signal and noise, then as a consequence of a statistical independence of background fluctuations the signal/noise ratio when detecting the flare effect is increased through a coherent processing by at least a factor of $\sqrt{N}$, where $\sqrt{N}$ is the number of LOS.

The GPS technology provides the means of estimating TEC variations on the basis of phase measurements of TEC $I$ in each of the spaced two-frequency GPS receivers using the formula (Hofmann-Wellenhof et al., 1992; Calais and Minster, 1996)

$$
\begin{gathered}
I=\frac{1}{40.308} \frac{f_{1}^{2} f_{2}^{2}}{f_{1}^{2}-f_{2}^{2}}\left[\left(L_{1} \lambda_{1}-L_{2} \lambda_{2}\right)+\right. \\
+ \text { const }+n L]
\end{gathered}
$$

where $L_{1} \lambda_{1}$ and $L_{2} \lambda_{2}$ are the increments of the radio signal phase path caused by the phase delay in the ionosphere (m); $L_{1}$ and $L_{2}$ stand for the number of complete phase rotations, and $\lambda_{1}$ and $\lambda_{2}$ are the wavelengths (m) for the frequencies $f_{1}$ and $f_{2}$ respectively; const is some unknown initial phase path (m); and $n L$ is the error in determining the phase path $(\mathrm{m})$.

Phase measurements in the GPS system are made with a high degree of accuracy where the error in TEC determination for 30-s averaging intervals does not exceed $10^{14} \mathrm{~m}^{-2}$, although the initial value of TEC does remain unknown (Hofmann-Wellenhof et al., 1992).

The data analysis was based on using the stations where the local time during the flare varied from 10 to $17 \mathrm{LT}$.

Primary data include series of slant values of TEC $I(t)$, as well as the corresponding series of elevations $\theta(t)$ and azimuths $\alpha(t)$. These data are calculated using our developed CONVTEC program which converts the GPS system standard RINEX-files on the Internet (Gurtner, 1993).

The determination of SID characteristics involves selecting continuous series of $I(t)$ measurements of at least a one-hour interval in length, which includes the time of the flare. Series of elevations $\theta(t)$ and azimuths $\alpha(t)$ of the LOS are used to determine the coordinates of subionospheric points. In the case under consideration, all results were obtained for elevations $\theta(t)$ larger than $30^{\circ}$.

The method of coherent summation of time derivatives of the series of variations of the «vertical» TEC value was employed in studying the ionospheric response to solar flares. Our choice of the time derivative of TEC was motivated by the fact this derivative permits us to get rid of a constant component in TEC variations; furthermore, it reflects electron density variations that are proportional to the flux of ionizing radiation.

The coherent summation of time derivatives of the series of variations of the «vertical» TEC value was made by the formula

$$
S d=\sum_{i=1}^{n} d I(t) / d t_{i} \times K_{i}
$$

where $n$ is the number of LOS. The correction coefficient $K_{i}$ is required for converting the slant 


\subsubsection{X-ray Flare C2.5}

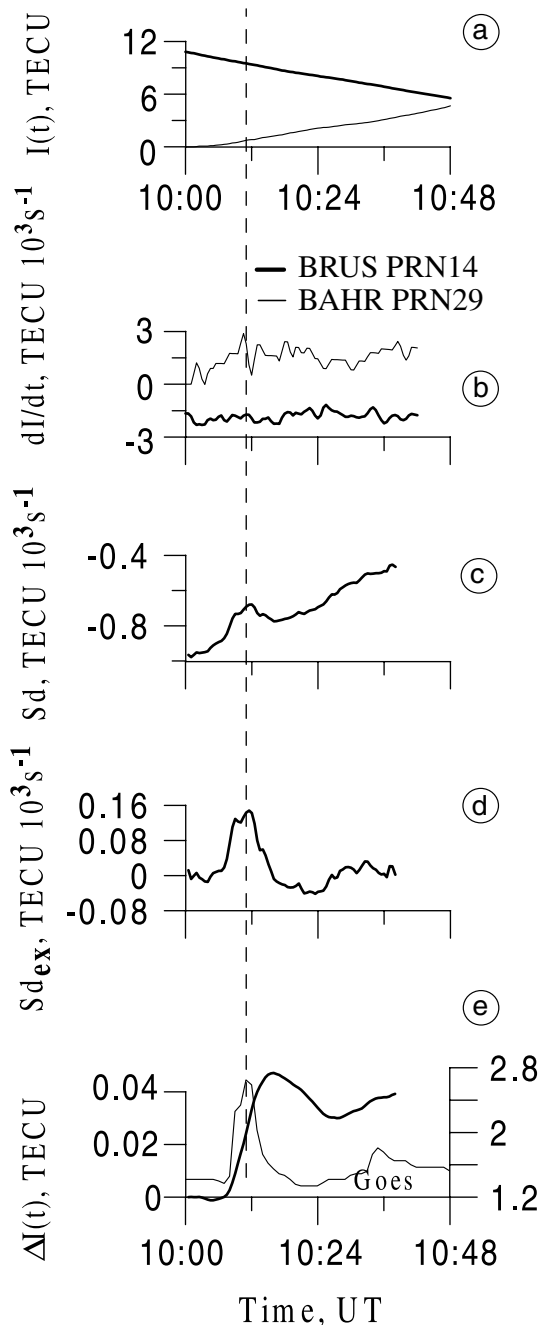

Fig. 1a-e. Faint solar flare July 29, 1999 (C2.5/SF, 10:11 UT, S16W11). The typical time dependencies of TEC variations for separate LOS (a) and their time derivatives (b) for stations BRUS (PRN14, thick line) and BAHR (PRN29, thin line); a normalized result of a coherent summation $S d$ of the time derivatives of the TEC variations for all LOS (c); the same curve (c), upon subtracting the trend determined as a polynomial of degree 3 on the time interval 10:0710:19 UT (d); mean integral increment of TEC (thick line) and soft X-ray emission flux (GOES-10) in the range 1-8 A (thin line) (e).
TEC to an equivalent «vertical» value (Klobuchar, 1986)

$$
K_{i}=\cos \left[\arcsin \left(\frac{R_{z}}{R_{z}+h_{\max }} \cos \theta_{i}\right)\right]
$$

where $R_{z}$ is Earth's radius; and $h_{\max }$ is the height of the ionospheric $F_{2}$-layer maximum.

Next the trend determined as a polynomial on a corresponding time interval is removed from the result (normalized to the number of LOS) of the coherent summation of the time derivatives. After that, the calculated time dependence $\left(S d_{e x}(t)\right)$ is integrated in order to obtain the mean integral TEC increment $\Delta I(t)$ on the time interval specified $\left[t_{1}-t_{2}\right]$.

$$
\Delta I(t)=\int_{t_{1}}^{t_{2}} S d_{e x}(t) d t .
$$

This technique is useful for identifying the ionospheric response to faint solar flares (of Xray class $\mathrm{C}$ ) when the variation amplitude of the TEC response to separate LOS is comparable to the level of background fluctuations.

\section{Ionospheric response to faint solar flare}

An example of a processing of the data for a faint solar flare July 29, 1999 (C2.5/ SF, 10:11 UT, S16W11) is given in fig. 1a-e. One hundred LOS were processed for the analysis of this event. Panels (a) and (b) present the typical time dependencies of TEC variations for separate LOS, and their time derivatives. The BRUS (PRN14, thick line) and BAHR (PRN29, thin line) stations are taken as our example. It is apparent from these dependencies that no response to the flare is distinguished in the TEC variations and in their time derivatives for the individual LOS, because the amplitude of the TEC response for the individual LOS is comparable to the level of background fluctuations.

A response to the solar flare is clearly seen in the time dependence (fig. 1c) which is a normalized result of a coherent summation $S d$ 
The response of the ionosphere to faint and bright solar flares as deduced from global GPS network data

29.07.99 X-ray Flare C2.2
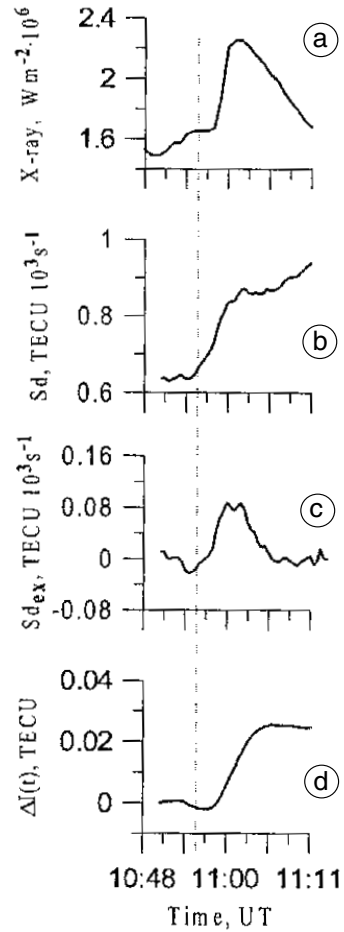

29.07.99 X-ray Flare C6.2
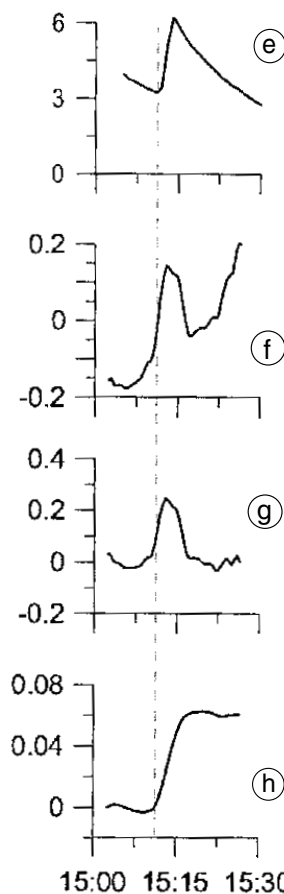

Time, UT

Fig. 2a-h. Faint solar flare July 29, 1999 (C2.2, 11:00 UT) (a-d); soft X-ray emission flux (GOES10 ) in the range 1-8 $\AA$ (a); normalized result of a coherent summation $S d$ of the time derivatives of the TEC variations for all LOS (b); the same curve (b), upon subtracting the trend determined as a polynomial of degree 3 on the time interval 10:4811:11 UT; mean integral increment of TEC (d). Analogous dependencies for faint solar flare July 29, 1999 (C6.2/SN, 15:14 UT, N25E53) on panels (e-h).

of the time derivatives of the TEC variations for all LOS. Upon subtracting the trend determined as a polynomial of degree 3 on the time interval 10:07-10:39 UT, the same curve (c) is presented in fig. $1 \mathrm{~d}$ as $S d_{e x}(t)$. Next the calculated time dependence was integrated over the time interval 10:07-10:39 UT to give the mean integral increment of TEC (fig. 1e, thick line). A comparison of the resulting dependence with the values of the soft X-ray

17.11.99 X-ray Flare C7.0

14.11.99 X-ray Flare C5.0
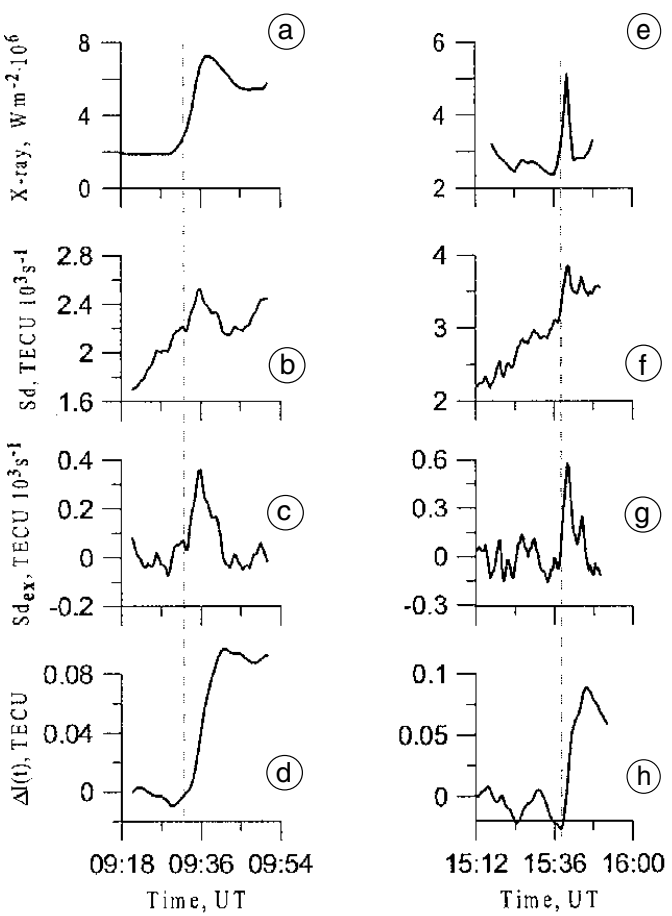

Fig. 3a-h. Faint solar flare November 17, 1999 (C7.0/ 1N, 09:38 UT, S15W53) (a-d); soft X-ray emission flux (GOES-10) in the range 1-8 $\AA$ (a); normalized result of a coherent summation $S d$ of the time derivatives of the TEC variations for all LOS (b); the same curve (b), upon subtracting the trend determined as a polynomial of degree 3 on the time interval 09:1809:54 UT (c); mean integral increment of TEC (d). Analogous dependencies for faint solar flare November 14, 1999 (C5.0, 15:40 UT) on panels (e-h).

emission flux (GOES-10) in the range 1-8 (fig. 1e, thin line) reveals that it has a more flattened form, both in its rise and fall. A maximum in X-rays is about 6 min ahead of that in TEC.

Examples of the application of our technology for the analysis of the ionospheric response to faint solar flares are given in figs. $2 \mathrm{a}-\mathrm{h}$ and $3 \mathrm{a}-\mathrm{h}$. Figure $2 \mathrm{a}-\mathrm{h}$ gives the data processing results on TEC variations for solar 
flares: July 29, 1999 (C2.2, 11:00 UT) on panels a, b, c, d, and July 29, 1999 (C6.1/SN, 15:14 UT, N25E53) on panels e, $\mathrm{f}, \mathrm{g}$, h. Figure $3 \mathrm{a}-\mathrm{h}$ shows the results of a data processing of TEC variations for solar flares of November 17, 1999 (C7.0/1N, 09:38 UT, S15W53) on panels a, b, c, d, and November 14, 1999 (C5.0, 15:40 UT) on panels e, f, g, h. Based on the above examples, it is possible to follow a correlation between the peak power of the faint flares in the X-ray range and the TEC enhancement in the ionosphere. For class $\mathrm{C} 2$ flares (fig. 1a-e, fig. 2a-d), the respective amplitude of TEC response is about twice as small as that for class C5-C7 flares (fig. 3a-h, fig. 2e-h). For C2.2 (fig. 2a-d) and C5 flares (fig. 3e-h), information about their localization on the Sun is unavailable.

\section{Ionospheric response to bright solar flares}

An example of a processing of the data for the bright solar flare of July 14, 1998 (M4.6/ $1 \mathrm{~B}, 12: 59 \mathrm{UT}, \mathrm{S} 23 \mathrm{E} 20)$ is given in fig. 4a-e.

Fifty LOS were used in the analysis of this event. Figure $4 \mathrm{a}$ presents the time dependencies of hard X-ray emission (CGRO/Batse, $25-50 \mathrm{keV}$, thick line on panels a) and of the UV line SOHO/SUMMER $171 \AA$, thin line) in arbitrary units (Aschwanden et al., 1999). It should be noted that the time dependence of the UV $171 \AA$ line is more flattened, both in the rise and in the fall, when compared with the hard X-ray emission characteristic. The increase in the UV $171 \AA$ line starts by about 1.8 min earlier, and the duration of its disturbance exceeds considerably that of the hard Xray emission disturbance.

Panel (b) presents the typical time dependencies of the TEC variations for separate LOS. The AOML (PRN24, thick line) and ACSI (PRN18, thin line) stations are taken as examples. A response to the bright flare is clearly distinguished for separate LOS. The normalized sum $S d$ of the time derivatives of the TEC variations for all LOS is presented in fig. 4c; panel (d) plots the same curve (c), upon subtracting the trend determined as a polynomial of degree 3 on the time interval

\subsubsection{X-ray Flare M4.6}
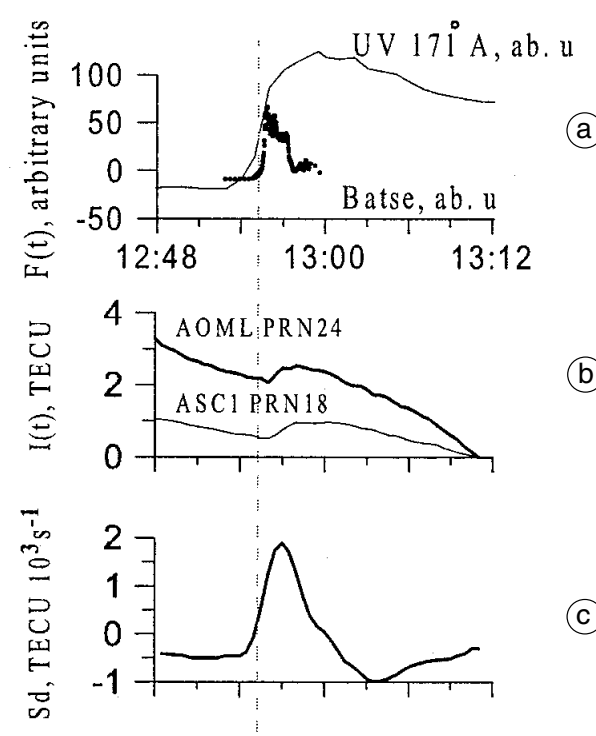

(C)

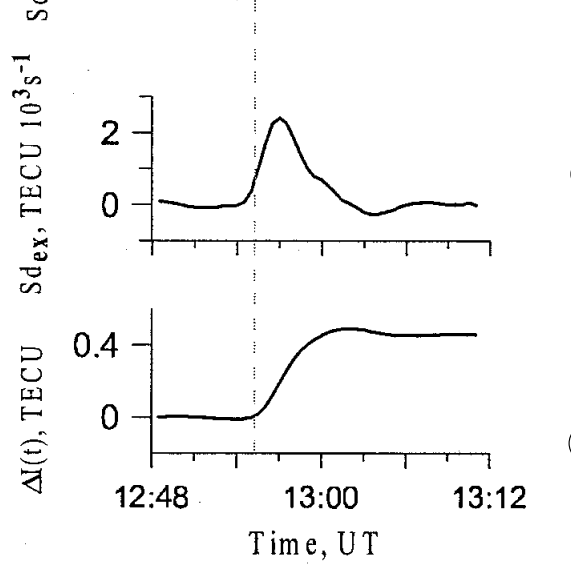

(b)

Fig. 4a-e. Bright solar flare July 14, 1998 (12:59 UT, M4.6/1B, S23E20). Time dependencies of hard $\mathrm{X}$-ray emission (CGRO/Batse, $25-50 \mathrm{keV}$, thick line) and of the UV line (SOHO/SUMMER $171 \AA$, thin line) in arbitrary units (a); typical time dependencies of the TEC variations for separate LOS for stations AOML (PRN24, thick line) and ACSI (PRN18, thin line) (b); the normalized sum $S d$ of the time derivatives of the TEC variations for all LOS (c); same curve (c), upon subtracting the trend determined as a polynomial of degree 3 on the time interval 12:48-13:12 UT (d); the mean integral increment of TEC (e). 
The response of the ionosphere to faint and bright solar flares as deduced from global GPS network data

Table I. Bright solar flares.

\begin{tabular}{cccccc}
\hline \hline $\mathrm{N}$ & $\begin{array}{c}\text { Data } \\
\text { dd.mm.yy }\end{array}$ & $\begin{array}{c}\text { Flare max time } \\
\mathrm{UT}\end{array}$ & $\begin{array}{c}\text { X-ray/optic } \\
\text { class }\end{array}$ & $\begin{array}{c}\delta T E C \\
T E C U\end{array}$ & $\begin{array}{c}\text { LAT CMD } \\
\text { degree }\end{array}$ \\
\hline 1 & 14.07 .98 & $12: 59$ & M4.6/1B & 0.50 & S23 E20 \\
2 & 19.08 .98 & $12: 42$ & M2.3/SF & 0.13 & N35 E78 \\
3 & 14.03 .99 & $01: 27$ & M2.1/1N & 0.3 & N13 E39 \\
4 & 04.04 .99 & $15: 25$ & M5.4/1F & 0.44 & N18 E72 \\
5 & 08.05 .99 & $14: 40$ & M4.6/1F & 0.33 & N23 W75 \\
6 & 29.07 .99 & $19: 36$ & M5.1/1N & 0.5 & N25 E51 \\
7 & 19.08 .99 & $16: 36$ & M3.0/2N & 0.7 & S31 E04 \\
8 & 27.08 .99 & $13: 07$ & M5.5/2N & 0.53 & S23 W09 \\
9 & 14.11 .99 & $16: 07$ & M5.6/- & 0.31 & N18 E60 \\
10 & 22.12 .99 & $19: 04$ & M5.3/1B & 0.66 & N24 E19 \\
11 & 28.12 .99 & $00: 48$ & M4.5/2B & 0.27 & N20 W56 \\
12 & 10.01 .00 & $13: 50$ & M3.3/2N & 0.13 & S13 E69 \\
13 & 23.03 .00 & $12: 14$ & M2.0/SF & 0.5 & N15 W69 \\
14 & 26.03 .00 & $17: 34$ & M2.3/SF & 0.26 & S12 W55 \\
15 & 08.04 .00 & $02: 40$ & M2.0/SF & 0.6 & S15 E26 \\
16 & 10.06 .00 & $16: 02$ & M5.2/3B & 0.5 & N22 W38 \\
17 & 23.06 .00 & $04: 07$ & M2.6/2B & 0.67 & N19 W30 \\
18 & 12.07 .00 & $18: 49$ & M5.7/2F & 0.30 & N16 W64 \\
19 & 14.07 .00 & $13: 52$ & M3.7/1N & 0.53 & N20 W08 \\
20 & 16.07 .00 & $02: 03$ & M5.5/1N & 0.08 & N09 E81 \\
21 & 18.07 .00 & $14: 19$ & M3.0/2N & 0.4 & S14 E15 \\
22 & 20.07 .00 & $20: 25$ & M5.0/1B & 0.67 & S15 W11 \\
23 & 19.09 .00 & $08: 26$ & M5.1/1N & 0.46 & N14 W46 \\
\hline
\end{tabular}

12:48-13:12 UT. Next the resulting time dependence was integrated in order to obtain the mean integral increment of TEC (fig. 4e). It should be pointed out that the time dependence of the mean integral increment of TEC has a more flattened form in the rise than the emission flux characteristics; however, the onset time of its increase coincides with that of hard X-ray emission, and is delayed by about $1.8 \mathrm{~min}$ with respect to the UV $171 \AA$ line.

A total of 23 events was processed (see table I). The class of X-ray flares was from $M$ 2.0 to $M$ 5.7. It was found that the mean TEC variation response in the ionosphere depends on the flare location on the Sun (central meridian distance, CMD) (fig. 5a).

\section{Discussion}

Our results are consistent with the findings reported by Donnelly (1969, 1971, 1976), Donnelly et al. (1986a), where a study of extreme UV (EUV) flashes of solar flares observed via SFD was made. In the cited references it was shown that the relative strength of impulsive EUV emission from flares decreases with increasing CMD and average peak frequency deviation is also significantly 


\section{Flares X-Ray M2.0 - M5.7}
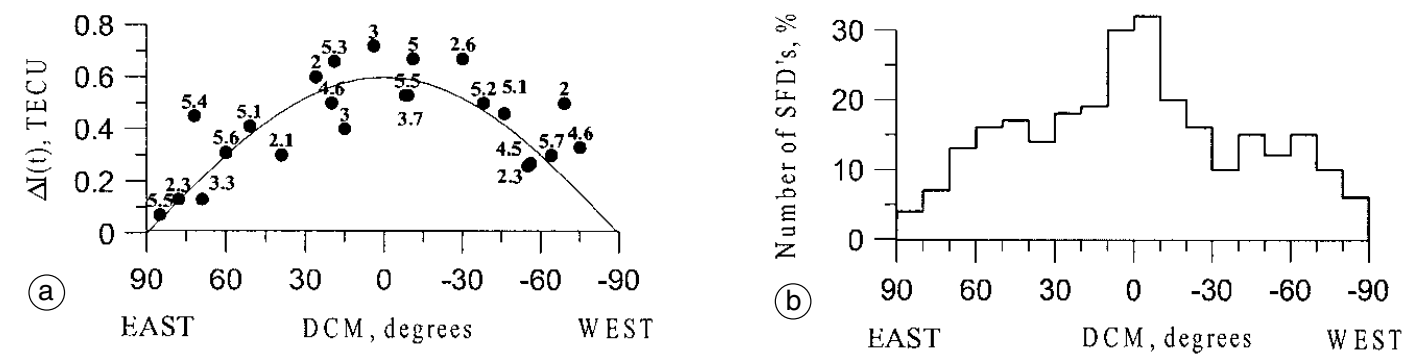

Fig. 5a,b. CMD dependence of the increment of the mean amplitude of the TEC response to solar flares (in the range of X-ray class $M 2.0-M 5.7$ (dots), $\cos (\mathrm{CMD})$ fitting curve for solar flares in the X-ray range $M 2.0-M$ 5.7 (solid lines) (a); percentage of $H \alpha$-flares accompanied by SFD's as a function of the central meridian distance of the $H \alpha$ flare (b).
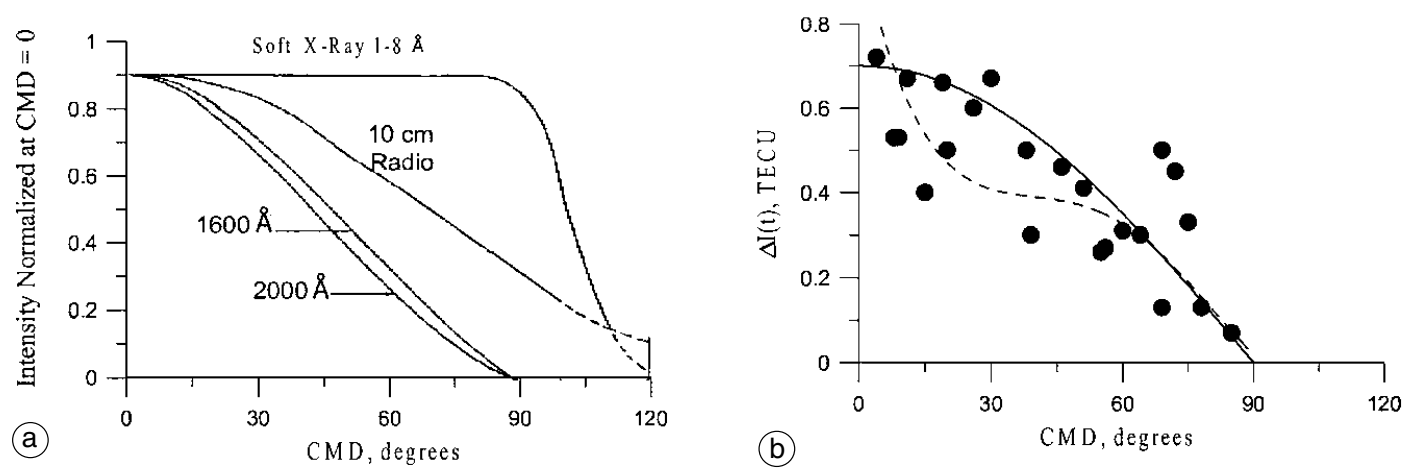

Fig. 6a,b. Average central meridian distance dependence based on (Mosher, 1979) for X-Ray (Riddle, 1969) and (Aschwanden et al., 1999) for F10, and (Samain, 1979) for the UV (a); modeling results on the SFD occurrence probability at the time of the solar flare as a function of the CMD (dashed lines) (Donnelly, 1976), values of the mean amplitude of the TEC response in the ionosphere to solar flares (dots), and cos(CMD) fitting curve for solar flares in the X-ray range $M 2.0-M 5.7$ (solid lines) (b).

lower for SFDs associated with $H \alpha$ flares at large CMD. Donnelly (1971) is of the opinion that percentage of $H \alpha$ flares with SFDs tends to decrease for large CMD of $H \alpha$ flare location (fig. 5b). Similar effects at the center and limb were observed in the ratio of EUV flux to the concurrent hard $\mathrm{X}$ ray flux (Kane and Donnelly, 1971). Using a fourth-order polynomial to fit the results in fig. $5 \mathrm{~b}$ with
CMD in degrees (Donnelly, 1976) gives

$$
\begin{gathered}
A(C M D) \simeq 1-0.0484 \cdot C M D+ \\
+0.001426 \cdot C M D^{2}-1.79 \cdot 10^{-5} \cdot C M D^{3}+ \\
+7.43 \cdot 10^{-8} C M D^{4} .
\end{gathered}
$$

Equation (5.1) implies that on the average the 
impulsive EUV emission is more than one order of magnitude weaker for flares near the solar limb than for flares at the central meridian. Donnelly (1976) has assumed that it results from the low-lying nature of the $10^{4} \mathrm{~K}-10^{6} \mathrm{~K}$ flare source region and from absorption of EUV emission in the surrounding cool nonflaring atmosphere. This conclusion is consistent with the findings reported by Donnelly and Puga (1990). In the cited reference it was found the empirical curves of the average dependence of active region emission on its CMD (fig. 6a) for several wavelengths (Donnelly et al., 1986a,b). Assume a quiet Sun plus one average active region that starts at the center of the backside of the Sun and rotates across the center of the solar disk with a 28-day period. Figure $6 \mathrm{~b}$ presents the result of a modeling of the SFD occurrence probability at the time of the solar flare as a function of CMD (dashed lines) in arbitrary units, as well as the values amplitude of the TEC response in the ionosphere to solar flares (in the range of X-ray class M 2.0-M 5.7, dots) as a function of CMD. The modeling used eq. (5.1). The figure suggests that the results of our measurements do not contradict the conclusions drawn by Donnelly (1976) Donnelly et al. (1986b) that the relative strength of impulsive EUV emission from flares decreases with increasing CMD. It should be noted that in the case of solar flares whose class is similar in X-ray emission, the dependence under study resembles $\cos (\mathrm{CMD})$ rather than a polynomial of degree 4 . The fitting $\cos (\mathrm{CMD})$ curve for solar X-ray $M$ 2.0-M 5.7 flares is plotted in figs. $6 \mathrm{~b}$ (solid line) and 5a (solid line).

\section{Summary}

This paper suggests a new method for investigating the ionospheric response to faint solar flares (of X-ray class $\mathrm{C}$ ) when the variation amplitude of the TEC response to individual LOS is comparable to the level of background fluctuations. The dependence of the TEC variation response amplitude on the flare location on the Sun is investigated. In the case of solar flares whose class is similar in X-ray emission, the dependence under study resembles $\cos (\mathrm{CMD})$. The high sensitivity of our method permits us to propose the problem of detecting, in the flare X-ray and EUV ranges, emissions of non-solar origins which are the result of supernova explosions. For powerful solar flares it is not necessary to invoke a coherent summation, and the ionospheric response can be investigated for each beam. This opens the way to a detailed study of the SID dependence on a great variety of parameters (latitude, longitude, solar zenith angle, spectral characteristics of the emission flux, etc.). With current increasing solar activity, such studies become highly challenging. In addition to solving traditional problems of estimating parameters of ionization processes in the ionosphere and problems of reconstructing emission parameters, the data obtained through the use of our method can be used to estimate the spatial inhomogeneity of emission fluxes at scales of the Earth's radius.

\section{Acknowledgements}

Authors are grateful to E.A. Kosogorov and O.S. Lesuta for preparing the input data. Thanks are also due to V.G. Mikhalkovsky for his assistance in preparing the English version of the manuscript. This work was done with support under RFBR grant of leading scientific schools of the Russian Federation No. 00-15-98509 and Russian Foundation for Basic Research (grants 99-05-64753, 00-05-72026 and 00-02-16819a), GNTP «Astronomy».

\section{REFERENCES}

AFRAIMOVICH, E.L. (2000): GPS global detection of the ionospheric response to solar flares, Radio Sci., 35 , 1417-1424.

AFraimovich, E.L., E.A. Kosogorov and L.A. LEONOVICH (2000): The use of the international GPS network as the global detector (GLOBDET) simultaneously observing sudden ionospheric disturbances, Earth, Planets, and Space, 52, 1077. 1082 .

Afraimovich, E.L., A.T. Altyntsev, E.A. Kosogorov, N.S. LARINA and L.A. LEONOVICH (2001a): Detecting of the ionospheric effects of the solar flares as deduced 
from global GPS network data, Geomagn. Aeron., 41 (2), 208-214.

Afraimovich, E.L., A.T. Altyntsev, E.A. Kosogorov, N.S. LARINA and L.A. LEONOVICH (2001b): Ionospheric effects of the solar flares of September 23, 1998 and July 29, 1999 as deduced from global GPS network data, J. Atmos. Sol.-Terr. Phys., 63 (17), 1841-1849.

AsCHWANDEN, M.J., L.. FLETCHER, C.J. SCHRIJVER and D. ALEXANDER (1999): Coronal loop oscillation observed with the transition region and coronal explorer Astrophys. J., 520, 880-894.

CALAIS, E. and J.B. Minster (1996): GPS detection of ionospheric perturbations following a Space Shuttle ascent, Geophys. Res. Lett., 23, 1897-1900.

DAVIES, K. (1980): Recent progress in satellite radio beacon studies with particular emphasis on the ATS-6 radio beacon experiment, Space Sci. Rev., 25, 357-430.

DAVIES, K. (1990): Ionospheric Radio (Peter Peregrinus, London), pp. 580.

DeshPANDE, S.D. and A.P. Mitra (1972): Ionospheric effects of solar flares, IV, electron density profiles deduced from measurements of SCNA's and VLF phase and amplitude, J. Atmos. Terr. Phys., 34, 255-261.

DoNNELLY, R.F. (1969): Contribution of X-ray and EUV bursts of solar flares to Sudden frequency deviations, J. Geophys. Res., 74, 1873-1877.

DONNELLY, R.F. (1971): Extreme ultraviolet flashes of solar flares observed via sudden frequency deviations: experimental results, Sol. Phys., 20, 188-203.

DONNELLY, R.F. (1976): Empirical models of solar flare Xray and EUV emission for use in studying their $E$ and $F$ region effects, J. Geophys. Res., 81, 4745-4753.

Donnelly, R.F. and L.C. PUGA (1990): Thirteen-day periodicity and the center-to-limb dependence of UV, EUV and X-ray emission of Solar activity, Sol. Phys. 130, 369-390.

DonNelly, R.F., H.E. HinteregGer and D.F. HEATH (1986a): Temporal variations of solar EUV, UV, and $10.830 \AA$ radiations, J. Geophys. Res., 91, 5567-5578.

DonNelly, R.F., L.C. PUGA and W.S. Busby (1986b): NOAA Tech. Memo. ERL ARL-146, NOAA ERL, Boulder, Colo.

HofMANN-Wellenhof, B., H. LichtenEgGer and J. Collins (1992): Global Positioning System: Theory and Practice (Springer-Verlag Wien, New York), pp. 327.

GURTNER, W. (1993): RINEX: The Receiver Independent Exchange Format Version 2.10I, http://igscb.jpl.nasa.gov:80/ igscb/data/format/rinex2.txt.
JONES, T.B. (1971): VLF Phase anomalies due to a solar Xray flare, J. Atmos. Terr. Phys., 33, 963-971.

KLOBUCHAR, J.A. (1986): Ionospheric time-delay algorithm for single-frequency GPS users, IEEE Trans. Aeros. Electron. Syst., 23 (3), 325-331.

KLOBUCHAR, J.A. (1997): Real-time ionospheric science: the new reality, Radio Sci., 32, 1943-1952.

KANE, S.R. and R.F. DONNELLY (1971): Impulsive hard Xray and ultraviolet emission during solar flares, Astrophys. J., 164, 151-163.

LIU, J.Y., C.S. CHIU and C.H. LIN (1996): The solar flare radiation responsible for sudden frequency deviation and geomagnetic fluctuation, J. Geophys. Res., 101, $10,855-10,862$.

MENDILLO, M. and J.V. EvANS (1974): Incoherent scatter observations of the ionospheric response to a large solar flare, Radio Sci., 9, 197-203.

Mendillo, M., J.A. Klobuchar, R.B. Fritz, A.V. DA Rosa, L. Kersley, K.C. Yeh, B.J. Flaherty, S. RANGASWAMY, P.E. SCHMID, J.V. EVANS, J.P. Schodel, D.A. Matsoukas, J.R. Koster, A.R. WEBSTER and P. CHIN (1974b): Behavior of the ionospheric $F$ region during the great solar flare of August 7, 1972, J. Geophys. Res., 79, 665-672.

MITRA, A.P. (1974): Ionospheric Effects of Solar Flares (D. Reidel, Norwell, Mass.), pp. 249.

MoshER, J.M. (1979): The height structure of solar active regions at X-Ray wavelengths as deduced from OSO8 limb crossing observations, Sol. Phys., 64, 109-115.

OHshio, M. (1971): Negative sudden phase anomaly, Nature, 229, 239-248.

RidDle, A.C. (1969): The quiet and slowly varying components of $9.1 \mathrm{~cm}$ radio emission during the silar minimum, Sol. Phys., 7, 434-439.

SAMAIN, D. (1979): Solar continuum data on absolute intensities, center to limb variations and laplace inversion between 1400 and $2100 \AA$, Astron. Astrophys., 74, 225-231.

SAO, K., M. YAMASHITA, S. TANAHASHI, H. JiNDOH and K. OHTA (1970): Sudden enhancements (SEA) and decreases (DSA) of atmospherics, J. Atmos. Terr. Phys., 32, $1567-1571$

STONEHOCKER, G.H. (1970): Advanced telecommunication forecasting technique in AGY, 5th., Ionospheric forecasting, AGARD Conf. Proc., 29, 27-31.

THOME, G.D and L.S.WAGNER (1971): Electron density enhancements in the $E$ and $E$ regions of the ionosphere during solar flares, J. Geophys. Res., 76, 6883-6895. 